\title{
The role of cognition for speech-in-noise perception: Considering individual listening strategies related to aging and hearing loss
}

DOI:

10.1177/0165025420914984

\section{Document Version}

Accepted author manuscript

Link to publication record in Manchester Research Explorer

Citation for published version (APA):

Heinrich, A. (2020). The role of cognition for speech-in-noise perception: Considering individual listening strategies related to aging and hearing loss. International Journal of Behavioral Development/I J B D, 1-7.

https://doi.org/10.1177/0165025420914984

\section{Published in:}

International Journal of Behavioral Development|I J B D

\section{Citing this paper}

Please note that where the full-text provided on Manchester Research Explorer is the Author Accepted Manuscript or Proof version this may differ from the final Published version. If citing, it is advised that you check and use the publisher's definitive version.

\section{General rights}

Copyright and moral rights for the publications made accessible in the Research Explorer are retained by the authors and/or other copyright owners and it is a condition of accessing publications that users recognise and abide by the legal requirements associated with these rights.

\section{Takedown policy}

If you believe that this document breaches copyright please refer to the University of Manchester's Takedown Procedures [http://man.ac.uk/04Y6Bo] or contact uml.scholarlycommunications@manchester.ac.uk providing relevant details, so we can investigate your claim.

\section{OPEN ACCESS}


THE ROLE OF COGNITION FOR SPEECH-IN-NOISE PERCEPTION: CONSIDERING INDIVIDUAL LISTENING STRATEGIES RELATED TO AGING AND HEARING LOSS

Antje Heinrich

The University of Manchester, Oxford Rd, Manchester, M13 9PL, UK.

Email: antje.heinrich@manchester.ac.uk

ORCID: $\underline{0000-0002-5940-622 X}$ 


\section{Abstract}

Listening to speech in a noisy background is difficult for everyone. While such listening has historically been considered mainly in the context of auditory processing, the role of cognition has attracted considerable interest in recent years. This has been particularly true in the context of lifespan research and the comparison of younger and older listeners. This article will discuss three factors that are important to consider when investigating the nature of cognitive involvement in speech-in-noise (SiN) perception: 1) the listening situation; 2 ) listener variables; 3) the role of hearing aids. I argue that a similar level of intelligibility can be achieved with the support of very different skills, or listening strategies, depending on the listening situation and listener. Age as a variable is particularly instructive for this type of research as it is accompanied by auditory as well as cognitive changes. As age-related changes are not uniform, using aging as a tool for the investigation can increase the opportunity to see individual differences in contributing processes and resulting compensation strategies. In addition to highlighting different interaction effects between hearing and cognition, I will argue that our conceptual understanding of the underlying processes can only be furthered if the selection of cognitive tests and experimental procedures in speech-in-noise studies follows accepted cognitive models, so that understanding can advance on a conceptual level without reliance on a particular test. Ultimately, a better understanding of the various listening strategies available to listeners and the conditions under which they are used may have theoretical as well as practical implications. Theoretically, it may help us better understand phenomena such as listening effort. Practically, it may guide us towards more effective diagnosis and intervention for listening difficulties in everyday life.

Keywords: Cognition, Speech-in-noise perception, listening strategies, age, hearing loss 


\section{Introduction}

Listening to speech, particularly under adverse conditions, requires both auditory processing and cognitive engagement. The role of cognition specifically has been of growing interest to speech and hearing scientists as well as clinicians as evidenced by a number of recent reviews (e.g., Dryden, Allen, Henshaw, \& Heinrich, 2017; Füllgrabe \& Rosen, 2016; Humes et al., 2012; Mattys, Davis, Bradlow, \& Scott, 2012). Besides reviewing and meta-analysing recent experimental evidence on sensory-cognitive interaction and integration, a number of frameworks have been suggested to integrate the available evidence (e.g., Pichora-Fuller et al., 2016; Rönnberg, Holmer, \& Rudner, 2019; Schneider, Pichora-Fuller, \& Daneman, 2010; Wingfield \& Tun, 2007). The most prominent among them is the Ease of Language Understanding (ELU) model (Rönnberg et al., 2019; Rönnberg et al., 2013), although other models exist (Schneider \& Pichora-Fuller, 2000; Schneider et al., 2010). The ELU primarily focusses on the roles of working memory and executive function for speech understanding under adverse listening conditions. A main tenet of the model is that when speech quality is good, and a clear match between input and phonological representation in memory can be made, lexical access is rapid and resource-free, and speech perception is automatic with no requirement for top-down cognitive knowledge. However, when the input quality is poor, either due to internal (e.g., hearing loss) or external (e.g., background noise) factors, the match is not fast and resource-free and perception is not automatic. Instead the degraded auditory information has to be supported by linguistic or other knowledge, thus making the listening process conscious and effortful. Those interested in a more detailed description, analysis and critique of the ELU model are directed to Rönnberg and colleagues original papers (Rönnberg et al., 2019; Rönnberg et al., 2013) as well as Wingfield and colleagues' excellent discussion of the model (Wingfield, Amichetti, \& Lash, 2015). Of particular importance for the current paper is the fact that Wingfield and colleagues discuss many of the cognitive concepts employed in the ELU model within the wider field of cognitive psychology. They suggest ways in which the ELU model can be productively connected to cognitive and linguistic models to further elucidate its details. 
One aspect of the listening experience that receives comparatively little attention in any of the models but might be instructive in understanding the variable relationship of cognition to speech-innoise (SiN) perception is the nature of the listening situation. Investigating this relationship systematically is difficult because correlations between speech perception and cognition vary not only depending on the particular listening test and cognitive test but also depending on each test's (often unknown) retest reliability. One way of dealing with some of the variability in a study is to limit the variation in listening situation to only one dimension, e.g, to only change the target speech (e.g., Knight \& Heinrich, 2017) or the background noise (e.g., Zekveld, Rudner, Johnsrude, \& Rönnberg, 2013). Moreover, to increase the likelihood of seeing a meaningful change in correlations between two listening situations and cognitive function, changes in listening situation should be substantial rather than gradual. A disadvantage to both of these experimental strategies, i.e., extreme groups and reduced dimensionality, is that they make it more difficult to extend experimental results to real-life listening situations. Dryden and colleagues (2017) have suggested a multi-dimensional and finer-grained approach and investigated how previously published associations with cognition fit within it. I will discuss their results in the first section of this paper. Following this I will discuss how the differential contribution of cognition to SiN listening may depend on listener characteristics, with a special emphasis on age and hearing loss.

I aim to show that listening strategies can differ between different listening situations that are all classed, within their models, as SiN perception. Moreover, I will show that listening strategies can differ for identical listening situations depending on the characteristics of the listener. By discussing some of the seemingly contradictory evidence I will argue that in order to improve our understanding on a conceptual level, we will need to consider concepts developed within cognitive theories more closely. Finally, I aim to add to the discussion about ways in which this knowledge might be used to advance clinical assessment and rehabilitation. 


\section{The listening situation}

To understand what types of cognitive skills support intelligibility in a particular listening situation, it is important to consider what types of stimuli are presented and in what types of listening environments. Stimulus characteristics discussed here include the nature of target and background sounds, their relationship to each other, and the overall level of performance accuracy they generate.

An example of the differential involvement of cognition in listening was provided by a series of studies conducted by Heinrich and colleagues. In all of these studies the focus was on the nature of the target signal and how it affects cognitive involvement. Both studies showed that differences in cognitive performance only consistently predicted SiN perception when the task involved sentence perception, but not when it involved the perception of phonemes (Heinrich, Henshaw, \& Ferguson, $2015,2016)$. When the task was perception of single words the power of the predictive effect of cognition depended on the characteristics of the target words. Only the perception of words from a potentially unlimited set was predicted by individual differences in working memory (WM).

Conversely, when the words came from a limited set of nine digits, individual differences in cognition did not help to predict SiN performance. For sentence perception, a range of cognitive abilities including attention, inhibitory control and WM predicted performance, whereby the exact nature of the predictive relationship depended on whether the task comprised simple sentences in modulated noise or sentences masked by a single sentence.

Besides the nature of the target signal, the nature of the background sound also represents a stimulus dimension that determines the way in which cognition is engaged in listening. Most experimental results point to a stronger engagement of cognition when more complex maskers are present, but exactly how masker characteristics need to be constructed to engage particular cognitive processes is far from clear. For instance, Nüsse et al. (2018) used a number of fluctuating maskers of various types and combinations. Despite all maskers being acoustically complex, 
however, only the intelligible single speaker maskers in cafeteria noise showed a predictive relationship with cognitive abilities (lexical abilities). Similar results are reported by Zekveld et al. (2013). They investigated the association between cognitive function and SiN perception for single talker, fluctuating and stationary noise maskers, and found correlations with cognition (working memory) only in the single talker masker condition. Hence, experimental results suggest the following: first, the role of cognition for SiN perception differs depending on the type of background sound, with more complex sound probably engaging more cognitive processes; second, the particular cognitive process(es) engaged in listening depends on the specific characteristics of the background sound.

One more characteristic of the listening signal needs to be considered, namely the relationship between the two sounds. A number of studies have shown that the relative level of the two contributing signals, typically expressed as signal-to-noise ratio (SNR) is also important in determining which cognitive processes are engaged to achieve successful SiN perception. Lower SNRs tend to lead to lower levels of overall SiN performance. This is important to consider because overall levels of performance influence if and how cognition is engaged in perception, although the exact relationship between the two is far from clear. Some studies show a higher correlation between cognition and SiN perception at higher levels of overall intelligibility ( 80 versus 50 percent) (Larsby, Hällgren, \& Lyxell, 2011) while other studies show more complex patterns (Koelewijn, Zekveld, Festen, Rönnberg, \& Kramer, 2007).

Finally, to provide a further illustration of the complex nature of the relationship between listening situation and cognitive engagement, consider the study by Lunner and Sundewall-Thorén (2007) in which the authors found a correlation between SiN recognition in fluctuating noise (but not stationary noise) and working memory - a finding which is in partial contrast to the study by Zekveld et al. (2013). Two factors might have contributed to the divergent results. First, the studies used different WM tasks, which inadvertently may have tested different aspects of WM. Second, the 
studies used different groups of listeners: Lunner and Sundewall-Thorén tested older hearing aid users while Zekveld et al. tested young normal-hearing listeners. To what extent the characteristics of the cognitive predictor task and /or listener contributed to the differences in results between studies is unclear. I would like to argue that only a more theory-guided investigation of the relationship between these factors can help us disentangle these results. Given that cognitive theories have formalised many of the relationships between cognitive variables (e.g., Craik, 2016; Diamond, 2013; McGrew, 2009; Miyake \& Friedman, 2012) we need to consider their role more closely in cognitive hearing science.

\section{The listener}

Numerous listener characteristics may play a role in SiN perception. In the following section, I will discuss age, language status, educational status and cognitive abilities, of which age is the most widely studied listener characteristic in the context of SiN perception. Here I focus on differences found between listeners described as "young" adults, normally between the ages of 18 and 30 years old, and "older" adults, typically above the age of 60 years. I will concentrate on these "extreme" groups as they allow me to discuss the topic in the clearest way. However I would like to add that there is mounting evidence that the change in listening strategy already starts in middle age (Goossens, Vercammen, Wouters, \& van Wieringen, 2017). The listening performance of children will not be considered in this article. Interested readers are referred to Leibold and Buss (2019). Note that very little work exists that has explicitly addressed this age group in the context of comparing listening strategies with younger and older adults, even though the field as a whole and theoretical considerations in particular would benefit if the whole lifespan perspective were taken into account. 
Aging is accompanied by many changes, auditory, cognitive and linguistic. To what extent each change contributes to the common complaint by older listeners that they find SiN perception challenging is difficult to determine. With respect to auditory factors, some changes, such as reduced audibility of sound, are well known and currently form the basis for audiological intervention such as hearing aids. Other auditory changes have received less scrutiny but are probably just as important for SiN perception. They occur on many levels of the auditory system and include changes in the cochlea (Humes, 2008), in peripheral neural pathways (Kujawa \& Liberman, 2009) and in central pathways (Martin \& Jerger, 2005). For an excellent overview to auditory changes associated with aging, the reader is directed to Gordon-Salant and colleagues' (2010) collection of essays. Similarly, many changes in cognitive function occur with old age, an in-depth discussion of which in healthy and diseased aging can be found in Craik and Salthouse's (2015) handbook. Finally, changes in both domains are highly correlated (Baltes \& Lindenberger, 1997; Lindenberger \& Baltes, 1994), which has presented a considerable conundrum to aging researchers. In an attempt to understand this relationship, four hypotheses have been advanced: 1 ) sensory deprivation hypothesis, 2) information degradation hypothesis, 3) cognitive load hypothesis and 4) common-cause hypothesis. The first two hypotheses suggest that sensory decline causes cognitive decline with the time required for the effect to emerge being long versus short respectively. The third hypothesis suggests that decline in cognition causes decline in sensory processing. Finally, the common-cause hypothesis does not link sensation and cognition causally but instead suggests that a common factor underlies concurrent changes in both. For a more in-depth discussion see Schneider and Pichora-Fuller (2000).

Crucial for the current discussion is the question of what listeners do when faced with changes in either or both domains and how they adjust their listening strategies. It seems reasonable to assume that age-related changes in sensory and cognitive processing lead to changes in the relative contribution of each to SiN perception depending on what is most advantageous for a particular listener. This leads to group-level differences between younger and older listeners. A case in point is 
a series of studies in which a word-in-noise perception task was individually adjusted to produce the same overall accuracy level for both younger and older listeners (Heinrich \& Schneider, 2011; Murphy, Craik, Li, \& Schneider, 2000; Schneider, Avivi-Reich, Leung, \& Heinrich, 2016). Then, the accuracy with which the perceived words could be recalled from memory was measured and compared to recall accuracy in quiet. Results showed that in addition to the expected age-related memory deficit in quiet, memory for the words presented in noise was disproportionately impaired in older listeners compared to younger listeners despite comparable perceptual accuracy of the words. One way to interpret these results is to assume that older listeners had to invest disproportionately more attentional resources than younger listeners during the perception of the words. If the pool of attentional resources is limited (Kahneman, 1973) and shared between perception and memory then it is conceivable that the increased investment of resources during perception compromised subsequent memory performance. This interpretation is consistent with a meta-analysis by Füllgrabe and Rosen (2016) who found that working memory performance, while not a good predictor of SiN perception in young normal-hearing listeners, was a better predictor of SiN perception in older listeners, suggesting that working memory (and the associated use of attention) played an increasingly important role in SiN perception as listeners aged.

Apart from spending more attentional resources on SiN perception, older listeners have also been found to use semantic context to the same extent or a greater extent than their younger counterparts when given the opportunity. In a seminal paper, Pichora-Fuller at al. (1995) showed that older listeners did disproportionately well in SiN perception compared to their younger counterparts when the target sentences contained contextual information compared to when they did not. The authors suggest two potential interpretations for the result. Either, compared to younger adults, older listeners may allocate more resources to the perception process and thus benefit to a greater degree when additional information is available. Or, because younger and older listeners have to operate in identical SNRs in everyday listening situations which, in turn, means that older listeners tend to correctly perceive fewer words due to adverse age-related changes in 
hearing, they routinely have to compensate using contextual information. As a result, they may have more practice doing so (for a similar argument concerning $\mathrm{Cl}$ users see Dingemanse \& Goedegebure, 2019). In addition, given older adults' larger vocabulary (Verhaeghen, 2003) and increased confidence in using their linguistic knowledge (Kavé \& Halamish, 2015), older listeners may be more prepared to take qualified guesses about what word they thought they heard. All three types of behaviour would reflect a change in listening strategy resulting in a more signal-driven, bottom-up strategy for younger listeners and a more top-down, knowledge-driven strategy for older listeners (Avivi-Reich, Daneman, \& Schneider, 2014; Schneider, Avivi-Reich, \& Daneman, 2016). As aging is commonly accompanied by hearing loss, it can be difficult to disentangle whether the suggested changes in listening strategy are mainly due to age or to hearing. One way to investigate this question is to see whether listeners of comparable age but different amounts of hearing loss show different listening strategies. The next section will explore this questions.

Besides hearing loss, language competency and education have also been found to affect the strategies adopted by listeners. For instance, non-native listeners have been found to adjust their listening strategy to the aspects of linguistic knowledge they felt most comfortable with even if this is not the type of knowledge commonly used by native listeners. One illustration of this strategy is provided by Heinrich et al. (2010) who investigated the effectiveness of a particular acousticphonetic cue (English r-resonance) for SiN perception and showed that only native listeners were able to take advantage of this cue. Non-native listeners used different cues to achieve SiN perception in the same situation, and their choice of cue depended on their proficiency in the tested non-native language: non-native listeners with lower proficiency inappropriately attempted to transfer acoustic-phonetic knowledge of their native language to the non-native language; nonnative listeners with higher proficiency instead relied on lexical knowledge in their non-native language as a way of maximizing intelligibility. 
A final listener characteristic, which may impact upon listening strategies, is educational attainment. Although its role in modulating the relationship between cognition and SiN perception has yet to be systematically explored, Knight and Heinrich (2019) provide initial evidence to suggest that it may be an important factor. Inhibition, in addition to WM and attention, is often hypothesised to be important for SiN perception; however, Knight and Heinrich (2019) showed that only listeners with lower educational attainment showed the expected link between better inhibition scores and better SiN perception. In summary, the way in which people listen depends not only on the nature of the listening situation but also on listener characteristics including age, hearing loss, language status and educational attainment.

\section{The effect of hearing aid use}

Apart from considering age in the context of "normal" hearing, i.e. in the absence of clinical loss, age also plays an important role in the context of hearing aids as most hearing aid wearers are of older age. In fact, over 70 percent of people over the age of 70 have a clinical hearing loss (Action on Hearing Loss, 2019), which is most commonly treated by fitting hearing aids. Hearing aids amplify sound based on the hearing loss profile of the listener. In the UK alone over 4 million people wear hearing aids. Hearing aids (HA) are very likely to affect the way in which people listen. While we know a lot about how individual differences in cognition can affect the extent to which listeners derive benefit from HA use (for a review, see Souza, Arehart, \& Neher, 2015), it is less clear how HA use affects listeners' reliance on cognitive function to achieve SiN perception. A number of studies have suggested that differences in benefit derived from HA use can be explained by differences in working memory (Foo, Rudner, Ronnberg, \& Lunner, 2007; Lunner, 2003; Lunner, Rudner, \& Ronnberg, 2009; Ng et al., 2014; Rudner, Ronnberg, \& Lunner, 2011). However, given that we know that SiN listening in younger and older listeners without HAs relies on a broad range of cognitive abilities, and that the particular combination of abilities depends both on the cognitive make-up of 
the listener and the particulars of the listening situation, it seems unlikely that for this particular listener group WM is the only defining cognitive ability. On the other hand, to date it is not known what these other abilities might be, as most studies so far have focussed on the relationship between HA listening and WM.

One notable exception is Nüsse et al. (2018) who investigated cognitive measures beyond working memory, in particular attention and lexical knowledge. When comparing two groups of older adults (non-HA users with better hearing and HA users with poorer hearing) they found that their measures of cognition only predicted SiN perception in the group of relatively better-hearing non-HA users. In the HA user group, amount of hearing loss was the only predictor of SiN perception. Superficially, this result contrasts with the results of the research that has shown WM to be an important predictor (Ng et al., 2014; Rudner, Foo, Rönnberg, \& Lunner, 2009) but it is important to note that the predictive effect was either shown for new HA users (Ng et al. (2014) or for fairly new users (after a 9 week trial, Rudner et al., 2009). Ng and colleagues also showed that the strength of the relationship weakened over time and was no longer significant at six months post-fitting. In Nüsse et al.'s study all HA users had worn their HA for at least one year, and this difference in time interval between HA fitting and testing might explain the contradicting results. However, even if the results of these two studies can be reconciled, they still contrast with the plethora of studies in the literature that report a predictive relationship between individual differences in working memory and SiN perception in HA users (Souza et al., 2015). To further complicate matters, it is also important to keep in mind that just as with normal-hearing listeners, the predictive power of cognitive function interacted with the particular listening situation and was stronger for more complex (modulated) noise (Lunner \& Sundewall-Thorén, 2007). In summary, the way in which cognitive function interacts with HA use and what role the underlying hearing loss plays is still not well understood. WM may play a role; if and how this role differs from non-HA users, however, is not clear. Moreover, how other cognitive functions such as inhibition, attention, phonological and 
lexical processing as well as general comprehension are engaged in listening has not been explored in great detail.

\section{Discussion}

Speech perception in noise is a complex task and requires the involvement of bottom-up auditory as well as top-down cognitive processes. The realisation of the central role of cognition for SiN perception has led to the development of a new field of research, cognitive hearing science (Arlinger, Lunner, Lyxell, \& Pichora-Fuller, 2009). While the fact that cognition can play a pivotal role is now well established, we still know comparatively little about what this role entails in particular situations. Based on a brief overview of the literature, I would suggest the following considerations.

First, I have shown that the role and extent of cognitive involvement in SiN perception depends on the particular listening situation. Aspects of the listening situation that affect the type and extent of cognitive involvement include the nature of the target signal, the nature of the background sound, the relationship between target and background, and the overall difficulty level. Similarly, listener characteristics such as age, language status, educational status, cognitive abilities, and hearing status affect the nature and extent of cognitive engagement. Aging is a particularly useful dimension to consider when trying to understand listening strategies because it not only leads to group differences between younger and older adults but also increases the variability and individuality of listeners and more likely leads to a greater variety in listening strategies.

While WM undoubtedly plays an important role for SiN perception, it is also clear that WM cannot be the only cognitive function involved. A more comprehensive assessment of cognitive function and its relationship to listening in various situations is needed. However, to make such an assessment most effective, it has to be embedded within standard cognitive theory. Only when we understand how the cognitive skills we assess relate to each other, can we fully understand how they relate to 
SiN perception. An application of the knowledge, both theoretical and experimental, available in cognitive science could help us develop theoretically based predictions about which cognitive functions are involved in SiN listening, and under what conditions. Studies now increasingly consider the role of cognitive functions other than WM for SiN perception, including inhibition (Stenbäck, Hällgren, \& Larsby, 2016), attention (Heinrich et al., 2015), response control (Heinrich et al., 2016), vocabulary and language comprehension (Schneider, Avivi-Reich, \& Daneman, 2016; Schneider, Avivi-Reich, Leung, et al., 2016). Understanding the relationship of these concepts to one another and relating them systematically to different listening situations would greatly increase the potential clinical impact of this research. It could allow us to understand which cognitive functions individual listeners engage in the listening situations they encounter most often in their daily lives or find most important to master in the context of a fulfilling life. Based on this, and understanding of the influence of the characteristics of the individual listener, we could then develop suitable interventions and offer targeted training.

Second, an improved understanding of the role of cognitive function in SiN perception would allow us to better understand how this role changes with HA use. A more systematic understanding could be a stepping stone towards developing specific and targeted interventions and training programmes for new and experienced HA users. It could also lead to algorithm selection to best fit the individual needs of the listener. In line with Meister (2017), I suggest that the connection between cognition and speech perception can create perspectives for individualized treatment Third, listeners in different age groups use different listening strategies to achieve the same level of SiN intelligibility (Avivi-Reich, Jakubczyk, Daneman, \& Schneider, 2015; Schneider, Avivi-Reich, \& Daneman, 2016). I would like to posit that this mechanism is likely to occur in a gradual manner across all listeners and is driven by an individual's personal mixture of cognitive skills, knowledge and sensory ability. I would also like to highlight that while we normally concentrate on a small number of listening characteristics and tend to consider them in isolation, it is more likely that a number of 
these characteristics interact, sometimes compensating for, and at other times interfering with, each other. How these interactions occur, and which cognitive functions interact in which situations, still largely remains to be understood.

\section{Acknowledgment}

This work was supported by the NIHR Manchester Biomedical Research Centre.

I would like to thank Adriana Hanulikova for continued encouragement, and Astrid van Wieringen, Sarah Knight and Helen Glyde for constructive comments on earlier versions of this manuscript. 


\section{References}

Arlinger, S., Lunner, T., Lyxell, B., \& Pichora-Fuller, M. K. (2009). The emergence of cognitive hearing science. Scandinavian Journal of Psychology, 50, 371-384. doi:10.1111/j.1467$9450.2009 .00753 . x$

Avivi-Reich, M., Daneman, M., \& Schneider, B. A. (2014). How age and linguistic competence alter the interplay of perceptual and cognitive factors when listening to conversations in a noisy environment. Frontiers in Systems Neuroscience, 8, 21. doi:10.3389/fnsys.2014.00021

Avivi-Reich, M., Jakubczyk, A., Daneman, M., \& Schneider, B. A. (2015). How age, linguistic status, and the nature of the auditory scene alter the manner in which listening comprehension is achieved in multitalker conversations. Journal of Speech, Language, and Hearing Research, 58(5), 1570-1591. doi:10.1044/2015_JSLHR-H-14-0177

Baltes, P. B., \& Lindenberger, U. (1997). Emergence of a powerful connection between sensory and cognitive functions across the adult life span: a new window to the study of cognitive aging? Psychology and Aging, 12(1), 12-21. doi:10.1037//0882-7974.12.1.12

Craik, F. I. M. (2016). Memory, attention and aging: Selected works by Fergus I.M. Craik. New York: Routledge.

Craik, F. I. M., \& Salthouse, T. A. (2015). The Handbook of Aging and Cognition (3rd ed.): Psychology Press.

Diamond, A. (2013). Executive functions. Annual Review of Psychology, 64, 135-168. doi:10.1146/annurev-psych-113011-143750

Dingemanse, J. G., \& Goedegebure, A. (2019). The important role of contextual information in speech perception in cochlear implant users and its consequences in speech tests. Trends in Hearing, 23, 1-16. doi:10.1177/2331216519838672

Dryden, A., Allen, H. A., Henshaw, H., \& Heinrich, A. (2017). The association between cognitive performance and speech-in-noise perception for adult listeners: A systematic literature review and meta-analysis. Trends in Hearing, 21, 1-21. doi:10.1177/2331216517744675 
Foo, C., Rudner, M., Ronnberg, J., \& Lunner, T. (2007). Recognition of speech in noise with new hearing instrument compression release settings requires explicit cognitive storage and processing capacity. Journal of the American Academy of Audiology, 18, 618-631.

Füllgrabe, C., \& Rosen, S. (2016). On the (un)importance of working memory in speech-in-noise processing for listeners with normal hearing thresholds. Frontiers in Psychology, 7(1268). doi:10.3389/fpsyg.2016.01268

Goossens, T., Vercammen, C., Wouters, J., \& van Wieringen, A. (2017). Masked speech perception across the adult lifespan: Impact of age and hearing impairment. Hearing Research, 344, 109-124. doi:10.1016/j.heares.2016.11.004

Gordon-Salant, S., Frisina, R. D., Fay, R. R., \& Popper, A. N. (2010). The Aging Auditory System. New York: Springer.

Heinrich, A., Flory, Y., \& Hawkins, S. (2010). Influence of English r-resonances on intelligibility of speech in noise for native English and German listeners. Speech Communication, 52, 10381055. doi:10.1016/j.specom.2010.09.009

Heinrich, A., Henshaw, H., \& Ferguson, M. A. (2015). The relationship of speech intelligibility with hearing sensitivity, cognition, and perceived hearing difficulties varies for different speech perception tests. Frontiers in Psychology, 6(782). doi:10.3389/fpsyg.2015.00782

Heinrich, A., Henshaw, H., \& Ferguson, M. A. (2016). Only behavioral but not self-report measures of speech perception correlate with cognitive abilities. Frontiers in Psychology, 7(576). doi:10.3389/fpsyg.2016.00576

Heinrich, A., \& Schneider, B. A. (2011). Elucidating the effects of ageing on remembering perceptually distorted word pairs. The Quarterly Journal of Experimental Psychology, 64(1), 186-205. doi:10.1080/17470218.2010.492621

Humes, L. E. (2008). Aging and Speech Communication. The ASHA Leader, 13, 10-33. 
Humes, L. E., Dubno, J. R., Gordon-Salant, S., Lister, J. J., Cacace, A. T., Cruickshanks, K. J., . . . Wingfield, A. (2012). Central presbycusis: a review and evaluation of the evidence. Journal of the American Academy of Audiology, 23, 635-666. doi:10.3766/jaaa.23.8.5

Kahneman, D. (1973). Attention and Effort. Englewood Cliffs, NJ: Prentice-Hall.

Kavé, G., \& Halamish, V. (2015). Doubly blessed: older adults know more vocabulary and know better what they know. Psychology and Aging, 30(1), 68-73. doi:10.1037/a0038669

Knight, S. L., \& Heinrich, A. (2017). Different measures of auditory and visual Stroop interference and their relationship to speech intelligibility in noise. Frontiers in Psychology, 8, 230. doi: 10.3389/fpsyg.2017.00230

Knight, S. L., \& Heinrich, A. (2019). Visual inhibition measures predict speech-in-noise perception only in people with low levels of education. Frontiers in Psychology, 9, 2779. doi:10.3389/fpsyg.2018.02779

Koelewijn, T., Zekveld, A. A., Festen, J. M., Rönnberg, J., \& Kramer, S. E. (2007). Processing load induced by informational masking is related to linguistic abilities. International Journal of Otolaryngology. doi:10.1155/2012/865731

Kujawa, S. G., \& Liberman, M. C. (2009). Adding insult to injury: Cochlear nerve degeneration after "temporary" noise-induced hearing loss. The Journal of Neuroscience, 29, 14077-14085. doi:10.1523/JNEUROSCI.2845-09.2009

Larsby, B., Hällgren, M., \& Lyxell, B. (2011). Working memory capacity and lexical access in speech recognition in noise. In T. Dau, M. L. Jepsen, J. C. Dalsgaard, \& T. Poulsen (Eds.), Proceedings of ISAAR 2011: Speech Perception and Auditory Disorders. 3rd International Symposium on Auditory and Audiological Research. Nyborg, Denmark: The Danavox Jubilee Foundation. Leibold, L. J., \& Buss, E. (2019). Masked Speech Recognition in School-Age Children. Frontiers in Psychology, 10(1981). doi:10.3389/fpsyg.2019.01981

Lindenberger, U., \& Baltes, P. B. (1994). Sensory functioning and intelligence in old age: a strong connection. Psychology and Aging, 9(3), 339-355. 
Lunner, T. (2003). Cognitive function in relation to hearing aid use. International Journal of Audiology, 42, S49-S58.

Lunner, T., Rudner, M., \& Ronnberg, J. (2009). Cognition and hearing aids. Scandinavian Journal of Psychology, 50, 395-403.

Lunner, T., \& Sundewall-Thorén, E. J. (2007). Interactions between cognition, compression, and listening conditions: Effects on speech-in-noise performance in a two-channel hearing aid. Journal of the American Academy of Audiology, 18, 539-552.

Martin, J. S., \& Jerger, J. F. (2005). Some effects of aging on central auditory processing. The Journal of Rehabilitation Research and Development, 42, 25-44. doi:10.1682/JRRD.2004.12.0164

Mattys, S. L., Davis, M. H., Bradlow, A. R., \& Scott, S. K. (2012). Speech recognition in adverse conditions: A review. Language and Cognitive Processes, 27(7-8), 953-978. doi:10.1080/01690965.2012.705006

McGrew, K. (2009). CHC theory and the Human Cognitive Abilities Project: Standing on the shoulders of the giants of psychometric intelligence research. Intelligence, 37(1), 1-10. doi:10.1016/j.intell.2008.08.004

Meister, H. (2017). Speech audiometry, speech perception, and cognitive functions. HNO, 65, 1-4. doi:10.1007/s00106-016-0250-7

Miyake, A., \& Friedman, N. P. (2012). The nature and organization of individual differences in executive functions: Four general conclusions. Current Directions in Psychological Science, 21(1), 8-14. doi:10.1177/0963721411429458

Murphy, D. R., Craik, F. I. M., Li, K. Z. H., \& Schneider, B. A. (2000). Comparing the effects of aging and background noise on short-term memory performance. Psychology and Aging, 15(2), 323-334. doi:10.1037/0882-7974.15.2.323

Ng, E. H. N., Classon, E., Larsby, B., Arlinger, S., Lunner, T., Rudner, M., \& Rönnberg, J. (2014). Dynamic relation between working memory capacity and speech recognition in noise during the first 6 months of hearing aid use. Trends in Hearing, 18, 1-10. 
Nüsse, T., Steenken, R., Neher, T., \& Holube, I. (2018). Exploring the link between cognitive abilities and speech recognition in the elderly under different listening conditions. Frontiers in Psychology, 9(678). doi:10.3389/fpsyg.2018.00678

Pichora-Fuller, M. K., Kramer, S. E., Eckert, M. A., Edwards, B., Hornsby, B. W., Humes, L. E., .. . Wingfield, A. (2016). Hearing impairment and cognitive energy: The framework for understanding effortful listening (FUEL). Ear and Hearing, 37, 5S-27S. doi:10.1097/AUD.0000000000000312

Pichora-Fuller, M. K., Schneider, B. A., \& Daneman, M. (1995). How young and old adults listen to and remember speech in noise. Journal of the Acoustic Society of America, 97(1), 593-608.

Rönnberg, J., Holmer, E., \& Rudner, M. (2019). Cognitive hearing science and Ease of Language Understanding. International Journal of Audiology, 58(5), 247-261. doi:10.1080/14992027.2018.1551631

Rönnberg, J., Lunner, T., Zekveld, A., Sörqvist, P., Danielsson, H., Lyxell, B., . . Rudner, M. (2013). The Ease of Language Understanding (ELU) model: Theoretical, empirical, and clinical advances. Frontiers in Systems Neuroscience, 7(31). doi:10.3389/fnsys.2013.00031

Rudner, M., Foo, C., Rönnberg, J., \& Lunner, T. (2009). Cognition and aided speech recognition in noise: specific role for cognitive factors following nine-week experience with adjusted compression settings in hearing aids. Scandinavian Journal of Psychology, 50, 405-418. doi:10.1111/j.1467-9450.2009.00745.x.

Rudner, M., Ronnberg, J., \& Lunner, T. (2011). Working memory supports listening in noise for persons with hearing impairment. Journal of the American Academy of Audiology. doi:10.3766/jaaa.22.3.4

Schneider, B. A., Avivi-Reich, M., \& Daneman, M. (2016). How spoken language comprehension is achieved by older listeners in difficult listening situations. Experimental Aging Research, 42(1), 31-49. doi:10.1080/0361073X.2016.1108749 
Schneider, B. A., Avivi-Reich, M., Leung, C., \& Heinrich, A. (2016). How age and linguistic competence affect memory for heard information. Frontiers in Psychology, 7(618). doi:10.3389/fpsyg.2016.00618

Schneider, B. A., \& Pichora-Fuller, M. K. (2000). Implications of perceptual deterioration for cognitive aging research. In F. I. M. Craik \& T. A. Salthouse (Eds.), The Handbook of Aging and Cognition (2nd ed., pp. 155-219). Mahwah, NJ: Erlbaum.

Schneider, B. A., Pichora-Fuller, M. K., \& Daneman, M. (2010). The effects of senescent changes in audition and cognition on spoken language comprehension. In S. Gordon-Salant, R. D. Frisina, A. N. Popper, \& R. R. Fay (Eds.), Springer Handbook of Auditory Research: The Aging Auditory System: Perceptual Characterization and Neural Bases of Presbycusis (pp. 167-210). New York, NY: Springer.

Souza, P., Arehart, K., \& Neher, T. (2015). Working memory and hearing aid processing: Literature findings, future directions, and clinical applications. Frontiers in Psychology, 6(1894). doi:10.3389/fpsyg.2015.01894

Stenbäck, V., Hällgren, M., \& Larsby, B. (2016). Executive functions and working memory capacity in speech communication under adverse conditions. Speech, Language and Hearing, 19(4), 218-226. doi:10.1080/2050571X.2016.1196034

Verhaeghen, P. (2003). Aging and vocabulary score: A meta-analysis. Psychology and Aging, 18(2), 332-339.

Wingfield, A., Amichetti, N. M., \& Lash, A. (2015). Cognitive aging and hearing acuity: modeling spoken language comprehension. Frontiers in Psychology, 6(684). doi:10.3389/fpsyg.2015.00684

Wingfield, A., \& Tun, P. A. (2007). Cognitive supports and cognitive constraints on comprehension of spoken language. Journal of the American Academy of Audiology, 18(7), 548-558. doi:http://dx.doi.org/10.3766/jaaa.18.7.3 
Zekveld, A. A., Rudner, M., Johnsrude, I. S., \& Rönnberg, J. (2013). The effects of working memory capacity and semantic cues on the intelligibility of speech in noise. The Journal of the Acoustical Society of America, 134, 2225-2234. doi:10.1121/1.4817926 